\title{
A Variable Neighborhood Particle Swarm Algorithm Based on the Visual of Artificial Fish
}

\author{
Yang $\mathrm{Kai}^{1{ }^{1, *}, \text { Yong-long } \mathrm{Jin}^{2} \text { and Tian Ying }}{ }^{1}$ \\ ${ }^{1}$ University of Science and Technology LiaoNing, LiaoNing, China \\ ${ }^{2}$ Technical Center of TangShan Iron \& Steel, HeBei Iron \& Steel Group, HeBei, China
}

\begin{abstract}
Inspired by the model of artificial fish school algorithm, a novel particle swarm optimizer with the increasing visual of artificial fish is presented after introducing the visual of artificial fish into particle swarm optimization. The neighborhood of each particle is dynamically changed through continually expand the visual of each particle. The local optimal strategy and the global optimal strategy are combined to enhance information sharing among particles. By testing six standard benchmark functions, experimental results show that the improved algorithm performs better than the traditional PSO and may avoid falling into the local optimum instead.
\end{abstract}

Keywords: Swarm Intelligence, Intelligence algorithm, Convergence, Optimization

\section{INTRODUCTION}

The PSO algorithm was first introduced by Eberhart and Kennedy in $1995[1,2]$, originated from the simulation of the foraging behavior observed in bird flock. Because of it's high simulation speed and the algorithm's principle is simple and easy to realize, the PSO algorithm has enjoyed widespread popularity. Now, the algorithm has been successfully used in neutral network training [3], function optimization, pattern recognition, data mining, etc.

Although the PSO algorithm has many advantages, it also exist some defects such as premature convergence like other global optimum algorithm. How to overcome the premature convergence in the PSO algorithm is a hot topic noticed by more academic investigator. Different topologies and neighborhood structure of particle swarm have great effect to the optimization result of the algorithm, a great number of improved algorithms have arisen at present. Suganthan proposed a new strategy that the variable GBEST in the PSO algorithm is replaced by LBEST where a local neighborhood size is gradually increased and finally extended to include all particles [4]. The paper [5] adopt a dynamic topology that start as fully connected and end up being a ring topology, so that the risk of getting trapped somewhere in the search space is reduced. Ni qing-jian proposed a new particle algorithm that adopt multi-cluster structure [6]. In the proposed structure, particles in one cluster use the fully connected form to share information with each other and clusters exchange their information through ring topology. Yang xuerong proposed a improved algorithm called mutineighborhood particle swarm algorithm that the first particle in each neighborhood is used to accept the global information and the other particles can only accept the neighborhood information [7]. Gao ying introduced the idea that the particles were divided into some subgroups through clustering and the local optimal strategy was applied in the subgroup [8]. The result show the superiority of the algorithm. A novel version of PSO based on dynamic topology was proposed by Wen wen [9]. In the improved algorithm a probability of each particle was computed before iteration and the neighborhood optimal position was chose according to the probability.

In the paper, the visual of artificial fish in the artificial fish algorithm is introduced into particle swarm optimization on the basis of associated documents research. A new improved algorithm based on varying visual of artificial fish is presented (PSO-AFIV). The neighborhood topology of each particle is dynamically increased through continually expand the visual of each particle and include all the other particles in the end. By testing six standard benchmark functions, experimental results show that the improved algorithm performs better than the traditional PSO and may avoid falling into the local optimum instead.

\section{PSO ALGORITHM AND AFS ALGORITHM}

\section{A. The Original PSO Algorithm}

In PSO, a particle $x_{i}=\left(x_{i 1}, x_{i 2}, \cdots, x_{i D}\right)$ represent a potential solution to a problem in an D-dimensional search space. The velocity for particle $\mathrm{i}$ is represented as $v_{i}=\left(v_{i 1}, v_{i 2}, \cdots, v_{i D}\right)$. Every particle keeps a record of the best position that it has ever visited. Such a record is called the particle's previous best position and denoted by 
$p_{i}=\left(p_{i 1}, p_{i 2}, \cdots, p_{i D}\right)$. The best particle among all the particles in the population is represented by $p_{g}=\left(p_{g 1}, p_{g 2}, \cdots, p_{g D}\right)$.

In every iteration, the position and velocity of each particle are updated by following two formulas:

$v_{i d}^{k+1}=v_{i d}^{k}+c_{1} r_{1}\left(p_{i d}-x_{i d}^{k}\right)+c_{2} r_{2}\left(p_{g d}-x_{i d}^{k}\right)$

$x_{i d}^{k+1}=x_{i d}^{k}+v_{i d}^{k+1}$

Where $i=1,2, \cdots, M ; d=1,2, \cdots, N$, and $M$ is the size of the swarm; $k=1,2, \cdots$, determines the iteration number; $c_{1}, c_{2}$ are two positive constants, called cognitive and social parameter respectively; $r_{1}, r_{2}$ are two random functions in the range $[0,1]$.

The particle velocity in formula (1) is composed of three parts: the first part represents the influence of the particle's current speed. The second part of the formula is the "cognition" part that represents the personal thinking of the particle itself. The third part is the "social" part that represents the collaboration among the particles.

\section{B. Modified Particle Swarm Optimizer}

Shi and Elberhart firstly introduce a new parameter, called inertia weight, into the original particle swarm optimizer in 1998[10]. The formula (1) is replace by the following form:

$v_{i d}^{k+1}=w v_{i d}^{k}+c_{1} r_{1}\left(p_{i d}-x_{i d}^{k}\right)+c_{2} r_{2}\left(p_{g d}-x_{i d}^{k}\right)$

Where

$w=\left(w_{1}-w_{2} \times \frac{\text { max iter }- \text { iter }}{\max \text { iter }}+w_{2}\right)$

This $w$ plays the role of balancing the global search and local search. When $w$ is small, the PSO is more like a local search algorithm and has strong local search ability. When $w$ is large, the current velocity has a great influence on the speed in the next iteration and the PSO has strong global search ability. A time decreasing inertia weight is introduced which brings in a significant improvement on the PSO performance. We called the particle swarm optimization algorithm with inertia weight the standard PSO and denoted by PSO-TVIW.

Generally, in population-based optimization methods, it is desirable to encourage the individuals to wander through the entire search space, without clustering around local optima, during the early stages of the optimization. On the other hand, it is very important to enhance convergence toward the global optima at the end of the search, to find the optimum solution efficiently. Asanga Ratnaweera propose a modified algorithm referred to as PSO-TVAC method by changing the acceleration coefficients $c_{1}, c_{2}$ with time [11]. i.e.

$$
\begin{aligned}
& c_{1}=\left(c_{1 f}-c_{1 i}\right) \times \frac{\text { iter }}{\max \text { iter }}+c_{1 i} \\
& c_{2}=\left(c_{2 f}-c_{2 i}\right) \times \frac{\text { iter }}{\max \text { iter }}+c_{2 i}
\end{aligned}
$$

where $, c_{1 f}, c_{1 i}, c_{2 f}, c_{2 i}$ are constants, iter is the current iteration number and maxiter is the maximum number of allowable iterations. It has been observed that the performance of the PSO-TVIW method is significantly improved for unmodal functions, but similar or poor for multimodal functions.

\section{Artificial Fish School Algorithm}

Recently, artificial fish school algorithm (AFSA) comes forth as a new evolutionary computation intelligent method, proposed by Dr. Li xiao-lei on the basis of imitation for fish behavior [12]. Artificial fish school algorithm is designed based on virtual vision of artificial fish. Biology vision system is an extremely complex system. It can quickly perceive lots of things in the environment and no other machines and program can be compared with it.

Let $X^{i}=\left(x_{1}^{i}, x_{2}^{i}, \mathrm{~L}, x_{n}^{i}\right)$ be the current state of an artificial fish and Visual be the vision range of the artificial fish. Assume that $X^{j}=\left(x_{1}^{j}, x_{2}^{j}, \mathrm{~L}, x_{n}^{j}\right)$ be a position in a moment within the perceived distance Visual. If the state in that position is better than current position, consider a step forward to that direction and arrive at the state $X_{n e x t}$. Otherwise, the artificial fish continue to patrol the other location within the field of view. This process can be expressed as:

$$
\begin{aligned}
& X^{j}=X^{i}+\text { Visual }^{*} \text { Rand }() \\
& X_{\text {next }}=X^{i}+\frac{X^{j}-X^{i}}{\left\|X^{j}-X^{i}\right\|} * \text { step }^{*} \text { Rand }
\end{aligned}
$$

The behavior of artificial fish optimization in AFSA include: random behavior, prey behavior, swarm behavior and follow behavior. The interconversion of these behaviors takes place constantly within the visual of artificial fish by continuous assessment, in order to achieve the purpose of looking for food or companions.

\section{IMPROVED PSO BASED ON INCREASING VISU- AL OF ARTIFICIAL FISH(PSO-AFIV)}

\section{A.Basic Description}

In this paper, the visual of artificial fish is firstly introduced into particle swarm optimization after inspired by the 
optimization process artificial fish applied within their visual. Every particle is assigned a vision with a limited range, denoted by visual. The other particles within visual of a given particle form the neighborhood of it. In the standard PSO, every particle is affected by both individual and population experience in solution space. In order to promote the capabilities of information exchange and sharing, a new component is added into the velocity formula which represents the attraction generated from the neighborhood optimum. The new formula is as follow:

$$
\begin{aligned}
& v_{i d}^{k+1}=w v_{i d}^{k}+c_{1} r_{1}\left(\alpha\left(p_{i d}-x_{i d}^{k}\right)+\right. \\
& \left.(1.2-\alpha)\left(p_{l d}-x_{i d}^{k}\right)\right)+c_{2} r_{2}\left(p_{g d}-x_{i d}^{k}\right)
\end{aligned}
$$

Where $\alpha$ is a random function in the range $[0,1]$ and $p_{l d}$ represents the best neighborhood optimum of a particle $x_{i}$ so far.

Although PSO algorithm can get a better solution than other evolutionary algorithms in a faster way, it does not necessarily carry out more accurate search as iteration times increases. This paper introduces an idea that the visual of particles is variable with iteration times increases. In the initial stage of the iteration, the variable Visual is set to 0 . That means the neighborhood of a particle only contain itself. As the iteration proceeds, the visual of particles increases gradually and the number of neighborhood particles belonging to a particle increases correspondingly, finally, contains all the particles in the population. The formula is described as follow:

Visual $=$ Visual_ $\max \times \frac{\text { iter }}{\max \text { iter }}$

Where Visual_max is a specified maximum of visual. In addition, in order to limit the particle velocity in the later iteration and make particles convergence to the global optimum solution, the constriction coefficient in [13] is adopted in this paper to control particle's trajectories.

\section{B. Mutation Operator}

Paper [14] introduce an effective method that identifies premature stagnation in PSO. So once premature stagnation happens, reinitialization will occur aimed at arbitrary onedimension of the current optimum. It is used to change the current searching trajectory so that particles can go out of the local optima and improve the accuracy of optimization.

Let Rmax be a predefined constant that represents the allowed maximum iteration number. That is to say, the optimal fitness value of the algorithm does not change through Rmax times of successive iterative computation. This means that the algorithm is likely to converge to a local optimum of the problem, then change arbitrary one-dimension of the current optimum randomly. i.e. choose any element and replace it by a random number within the prescribed limits so as to enhance the exploration ability for particles and escape from the local optimum for the algorithm.

$$
\begin{aligned}
& \text { If } \operatorname{fbest}(n)=f \operatorname{best}(n-R \max ) \text { then } \\
& d=\operatorname{rand}(D) \\
& p_{g d}=\operatorname{rand} \times\left(d_{\max }-d_{\min }\right)+d_{\min } \\
& \text { Endif }
\end{aligned}
$$

Where fbest(n) is the optimal fitness value of the whole population in $n$-th iteration, are lower and upper bounds of the d-dimension respectively. This paper applies such effective and convenient operator to the improved PSO.

\section{The Algorithm Steps}

The new algorithm can be summarized as follows:

Step 1: Initialize parameters: the population size $N$, the maximum iteration number LoopCount, the acceleration coefficients $c_{1}, c_{2}$, the max visual Visual_max, inertia weight $w$, Rmax, the constriction coefficient $\chi$, the max velocity $v_{\max }$.

Step 2: Initialize position and velocity of all the particles randomly in the $N$ dimension space.

Step 3: Evaluate the fitness value of each particle, and update the global optimum position $p_{g}$, the neighborhood optimum position $p_{l d}$ and the individual optimum position $p_{i}$.

Step 4: Update the visual of population according to formula (10). (4).

Step 5: Update the inertia weight according to formula

Step 6: Identify whether the algorithm has stagnated through successive iteration during Rmax times. If the algorithm has stagnated, initialize any one-dimension of the optimal position by mutation operator according to 3.2.

Step 7: For each particle, update particle velocity according formula (9), update particle position according formula (2).

Step 8: Determine the individual best fitness value. Compare the $p_{i}$ of every individual with its current fitness value. If the current fitness value is better, assign the current fitness value to $p_{i}$.

Step 9: Determine the best fitness value in each particle's neighborhood. If the current fitness value of a particle within one's visual is better than the $\mathrm{p}_{\mathrm{ld}}$, assign the current fitness value to $\mathrm{p}_{\mathrm{ld}}$.

Step 10: Determine the current best fitness value in the entire population. If the current best fitness value is better than the $\mathrm{p}_{\mathrm{g}}$, assign the current best fitness value to $p_{g}$.

Step 11: Repeat Step 4 - 10 until a stop criterion is satisfied or a predefined number of iterations are completed. 


\section{ALGORITHM TESTING}

In order to evaluate the methods described in the section 3 , the new improved algorithm (PSO-AFIV) described above along with several variations of PSO are tested on six optimization functions commonly used by researchers of PSO systems. The several variations of PSO compared with are the standard PSO (PSO-TVIW), the modified algorithm of PSO with mutation operation(PSO-PCIW), the PSO with adaptive acceleration coefficients (PSO-TVAC). Table $\mathbf{1}$ is parameters setting for the six functions. All the experiments were conducted using a population size of 60 , with each algorithm executed for a maximum of 1000 generations. The Schaffer function is 2 dimensional, all the others were in 30 dimensions.

\section{A. Benchmark Functions}

The following is functions that were used:

1.Griewank

$$
\begin{aligned}
& f(x)=\frac{1}{4000} \sum_{i=1}^{n} x_{i}^{2}+\prod_{i=1}^{n} \cos \left(\frac{x_{i}}{\sqrt{i}}+1\right), \\
& -100 \leq x_{i} \leq 100
\end{aligned}
$$

2.Rastrigin

$$
\begin{aligned}
& f(x)=\sum_{i=1}^{n}\left(x_{i}^{2}-10 \cos \left(2 \pi x_{i}\right)+10\right), \\
& -5.12 \leq x_{i} \leq 5.12
\end{aligned}
$$

3.Sphere

$f(x)=\sum_{i=1}^{n} x_{i}^{2},-100 \leq x_{i} \leq 100$

$$
\text { 4. Ackley }
$$

$$
\begin{aligned}
& f(x)=-20 \exp \left(-0.2 \sqrt{\frac{1}{n} \sum_{i=1}^{n} x_{i}^{2}}\right. \\
& \left.-\exp \left(\frac{1}{n} \sum_{i=1}^{n} \cos \left(2 \pi x_{i}\right)\right)\right)+20+e \\
& -32 \leq x_{i} \leq 32 \\
& \text { 5. RosenBlock }
\end{aligned}
$$

$$
\begin{aligned}
& f(x)=\sum_{i=1}^{n}\left[100\left(x_{i+1}-x_{i}^{2}\right)^{2}+\left(x_{i}-1\right)^{2}\right]-30 \leq x_{i} \leq 30 \\
& \text { 6. Schaffer } \\
& f(x, y)=\left(x^{2}+y^{2}\right)^{0.25}\left\{\sin ^{2}\left[50\left(\left(x^{2}+y^{2}\right)^{0.1}\right]+1.0\right\}\right. \\
& -10 \leq x_{i} \leq 10
\end{aligned}
$$

\section{B. Results and Discussion}

A linearly decreasing inertia weight is used which starts at 0.9 and ends at 0.4 , with $c_{1}=2, c_{2}=2$, Population size is set to be 60 . The upper bound of visual is 100 . Vmax is set to be half of the initialization ranges of each function. The acceleration coefficients $\chi$ is set as 0.729 . Rmax is set as 10 . A total of 20 runs for each experimental setting are conducted. The experimental results is shown in Table 2, Fig. (1-6).

From Table 2, it can be seen that the convergence accuracy and success rate of PSO-AFIV is higher than the other three algorithms. The new algorithm reaches the optimum point for functions Griewank and Rastringin in every test. The success rate of PSO-AFIV is $100 \%$ for each function, and obviously better than PSO-TVIW, PSO-PCIW and PSOTVAC. PSO-AFIV has high stability according to the mean and deviation in Table 2.

The average best fitness convergence curve of each algorithm on six benchmark functions are shown in Fig. (1-6). For contrast, the ordinate in figure adopt the form of logarithmic. As shown in Figs. (1, 2, 3, 4, 5, 6 and Table 2), the new PSO-AFIV algorithm outperforms the other variants of PSO algorithm on each of the benchmark problems on which the experiments have been conducted so far. In each case, the original PSO and variants algorithm performs well in initial iterations but fails to make further progress in later iterations.

For Fig. (1) and Fig. (2), the improved algorithm converges to the global minimum point quickly within the 600 th iteration, while the other algorithms are all falling into the local optimum instead. For the other four test functions as shown in Fig. (3-6), PSO-AFIV provides statistically superior results than the other PSO-variants in evidence. It can be seen from Fig. (5), although the convergence performance can be roughly the same as PSO-AFIV, it performs normally with respect to the other functions. A discourse on experimental studies show that the proposed variants PSO-AFIV significantly improve the searching capability of PSO with an enhanced convergence speed.

The significant improvement achieved by the PSO-AFIV algorithm can be attributed to the neighborhood interactions. In each iterations, each particle is updated by following three "best" values. The first one is the best solution it has achieved so far. The second is the best fitness obtained so far by any particle in the population. In addition to the above two components, a local best generated from topological neighbors of each particle is adopted to improve the population diversity.

The population diversity that is achieved can be demonstrated by the fact that the neighborhood of each particle is dynamically changed through continually expand the visual of each particle. The local optimal strategy and the global optimal strategy are combined to enhance information sharing among particles. In the initial stage of the iteration, the neighborhood of a particle only contains itself. As the iteration proceeds, the visual of particles increases gradually and the number of neighborhood particles belonging to a particle increases correspondingly, finally, contains all the particles in the population. This makes particles convergence to the global optimum solution effectively. 
Table 1. Parameters setting.

\begin{tabular}{|c|c|c|c|c|}
\hline Function & Dimention & Optimal Value & Generation & 1000 \\
\hline \hline Griewank & 30 & 0 & 1000 & 0.1 \\
\hline Rastrigin & 30 & 0 & 1000 & 50 \\
\hline Sphere & 30 & 0 & 1000 & 5 \\
\hline Ackley & 30 & 0 & 1000 & 100 \\
\hline Rosenbrock & 30 & 0 & 1000 & $1 \mathrm{e}-10$ \\
\hline Schaffer & 2 & 0 & \\
\hline
\end{tabular}

Table 2. Comparisions of the experimental results.

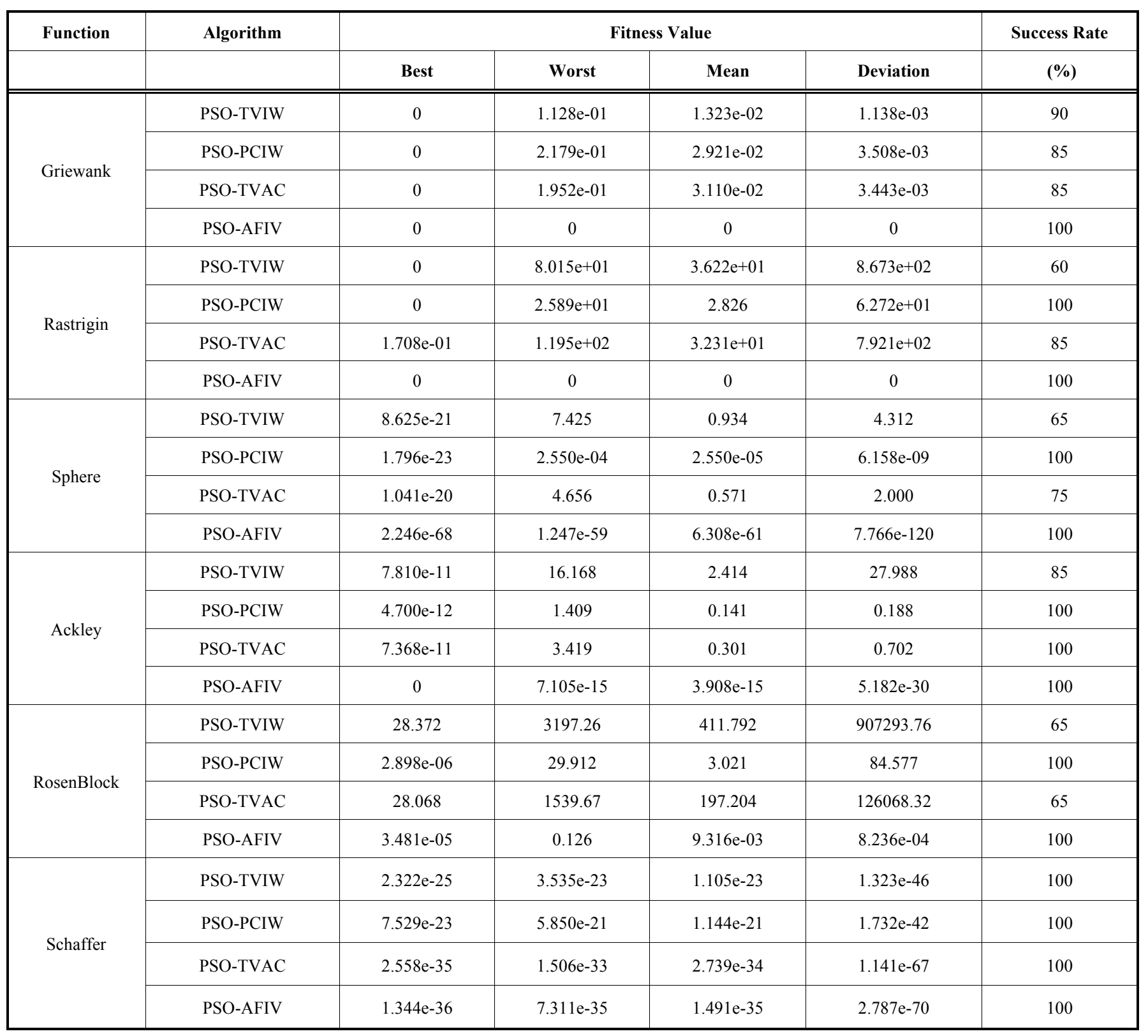




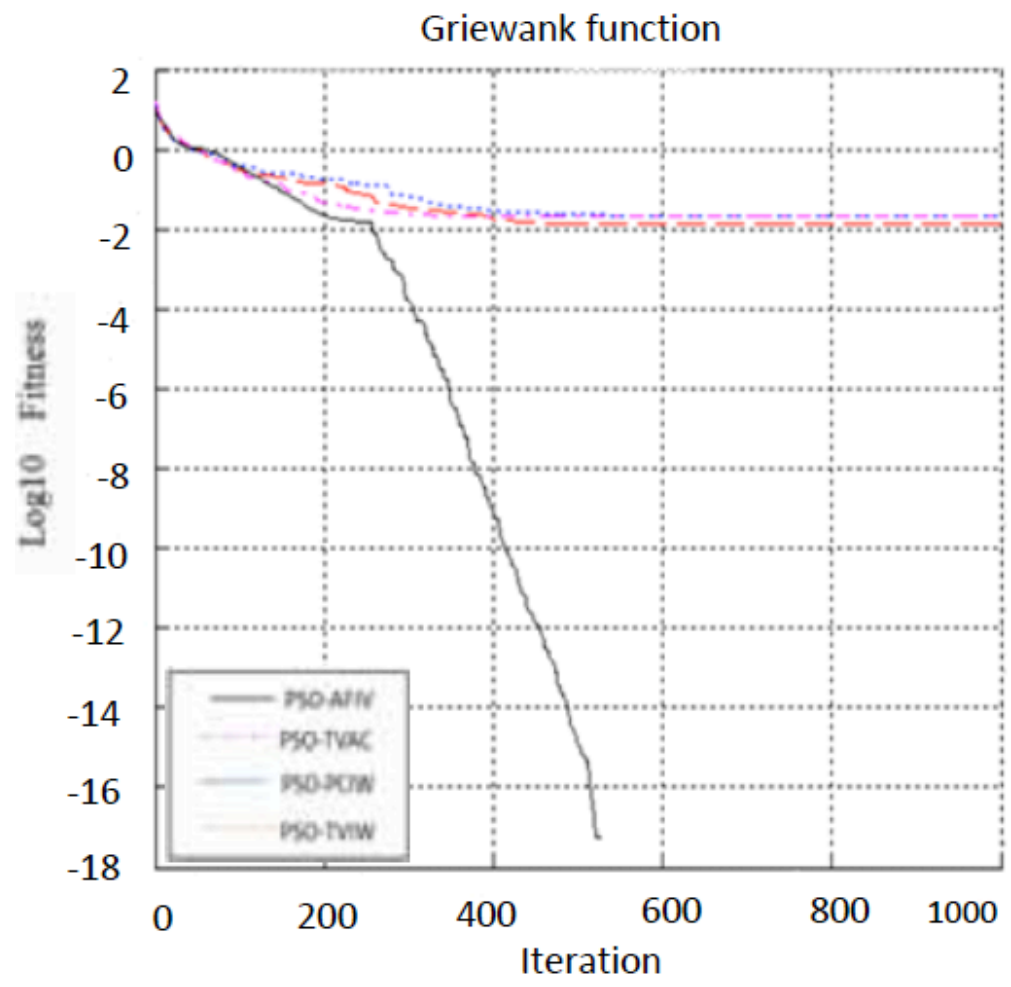

Fig. (1). Convergence graph for Griewank function.

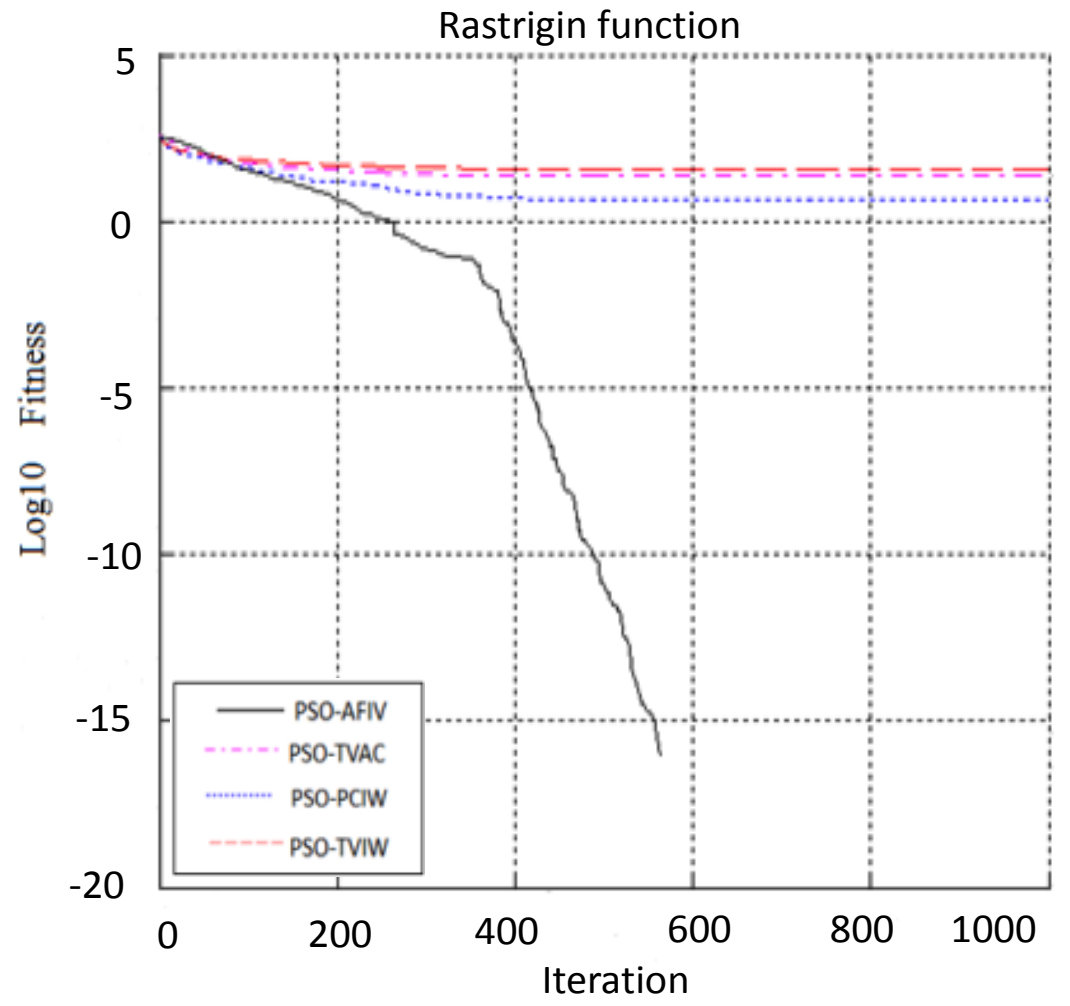

Fig. (2). Convergence graph for Rastrigin function. 


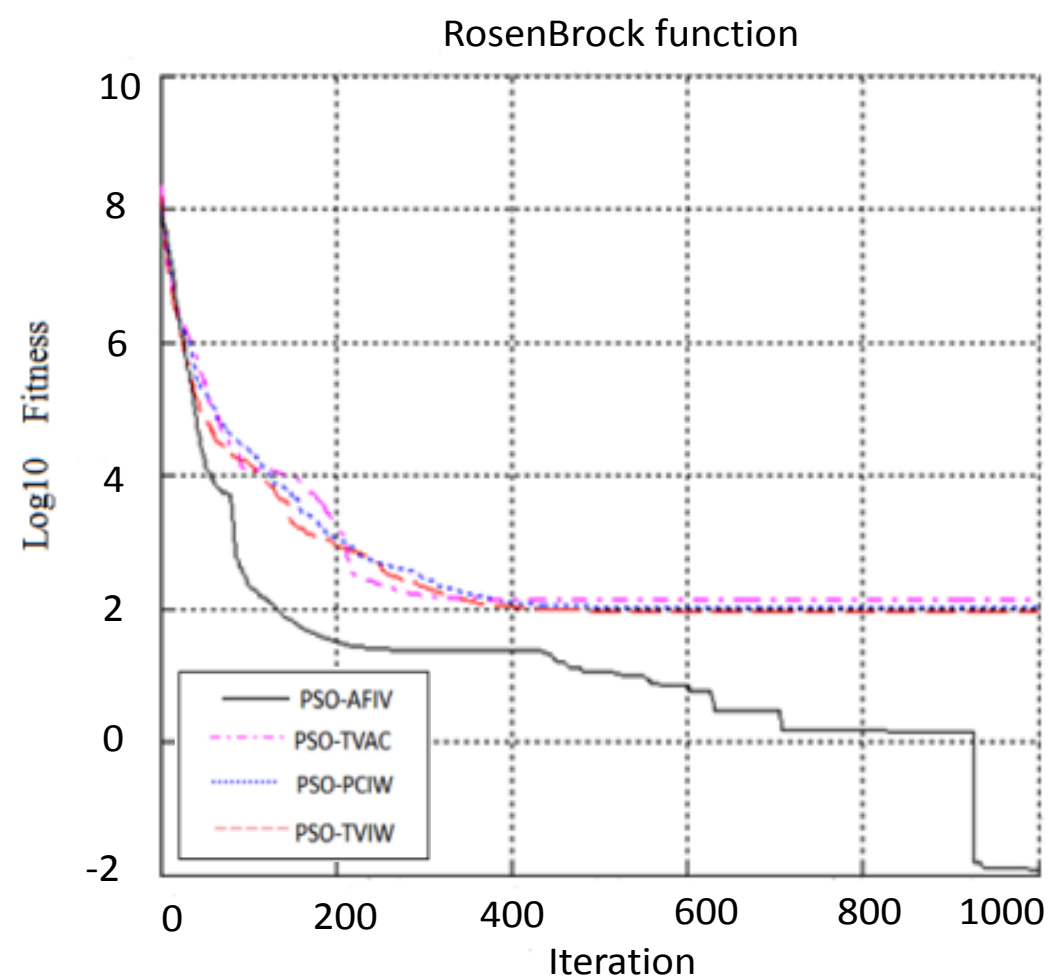

Fig. (3). Convergence graph for RosenBrock function.

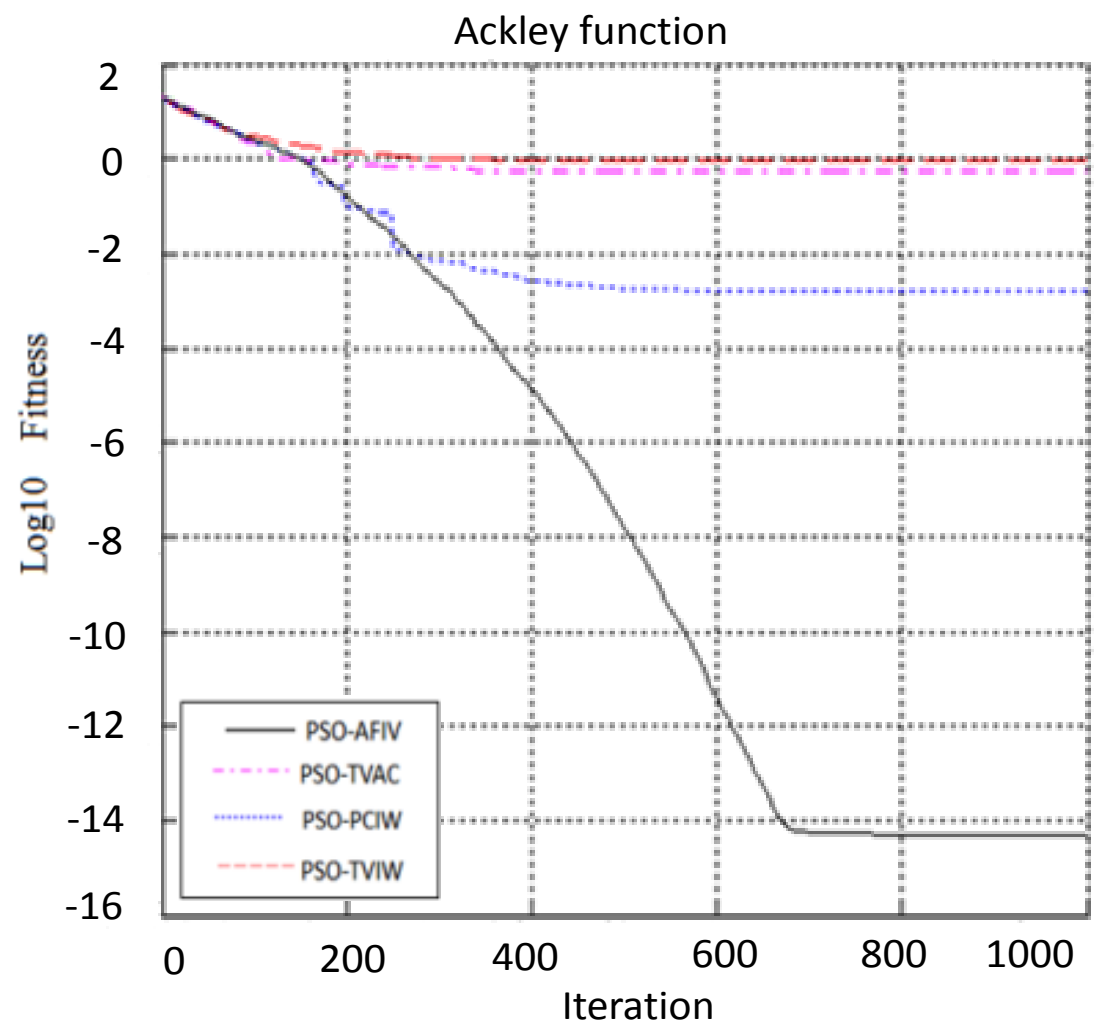

Fig. (4). Convergence graph for Ackley function. 


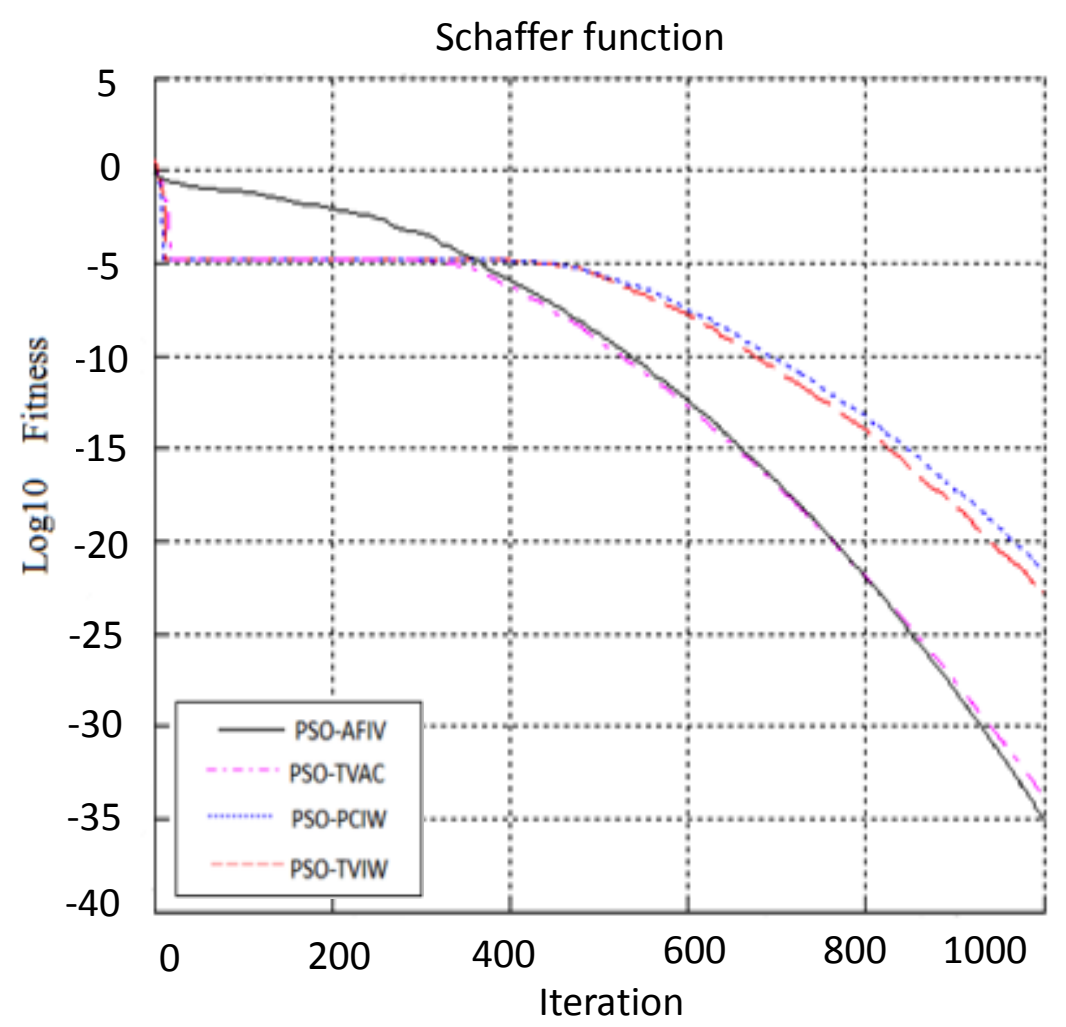

Fig. (5). Convergence graph for Schaffer function.

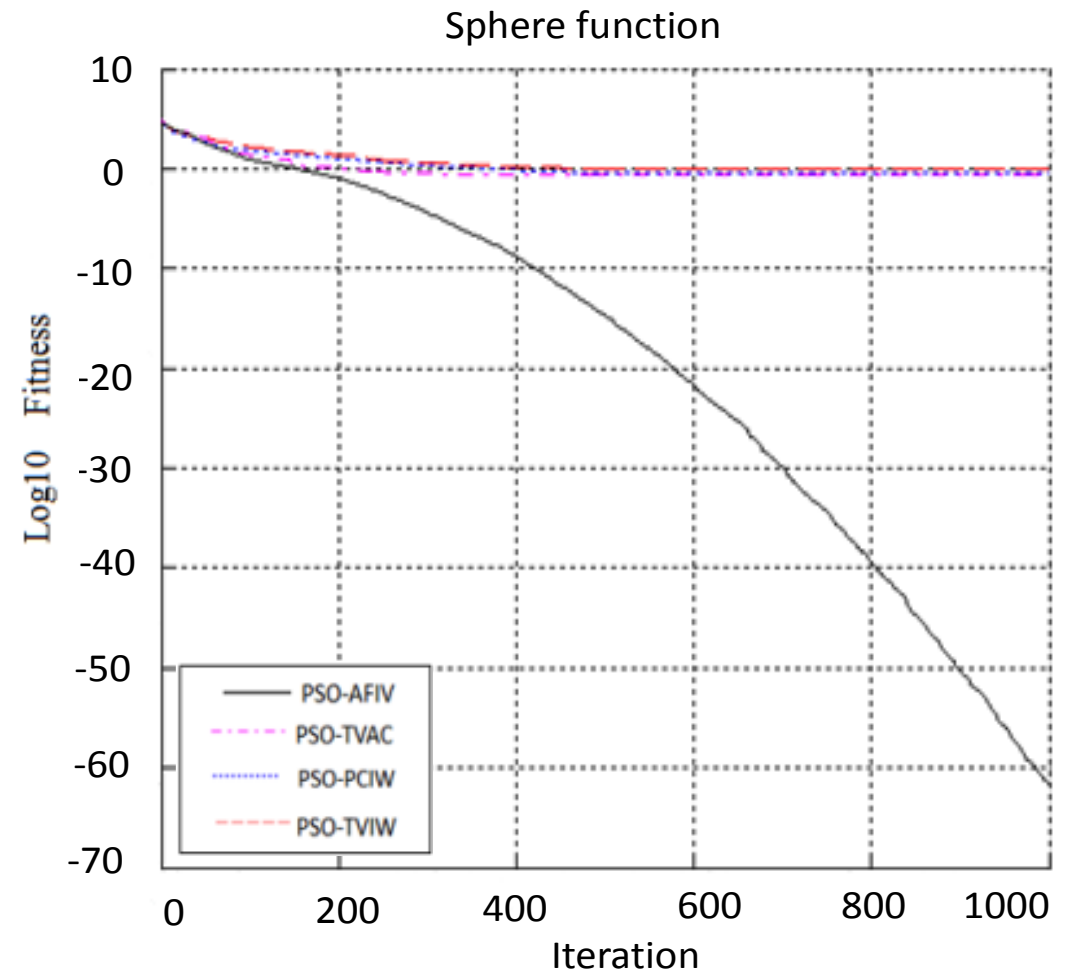

Fig. (6). Convergence graph for Sphere function. 


\section{CONCLUSION}

The neighborhood topology of particles is a very important factor for the performance of PSO. Different neighborhood topology may lead to very different results. So the researching and development of PSO attach more importance to the improvement of neighborhood topology. This paper introduce the visual of artificial fish school algorithm into PSO and presents a new improved variant based on incr easing visual of artificial fish. The neighborhood topology of each particle is dynamically increased through continually expand the visual of each particle. The hybrid algorithm kept population's diversity in the early iterations and converge to the global optimum quickly in the later period of iterations. The performance of the proposed algorithm was compared to other three variants of PSO with a set of unimodal functions and multimodal functions. The results showed that, the new algorithm significantly improve the searching capability of PSO and has faster convergence speed and better accuracy comparing to other strategies.

\section{ABOUT AUTHORS}

\section{Yang Kai,}

Current position, grades:

Associate professor in University of science and technology Liaoning

University studies:

Computer application technology in University of science and technology Liaoning

Scientific interest:

Data Mining, optimization algorithm

Publications $<$ number or main $>$ : 8 papers

Experience:

He received his master and bachelor degree majored in computer application technology in University of science and technology Liaoning in 2006 and 2003. Now he is a associate professor in University of science and technology Liaoning. His present research interests are data mining and soft computing.

\section{Yong-long Jin,}

Current position, grades:

Deputy director of technical center of Tangshan Iron \& Steel

University studies: Beijing

Steel metallurgy in University of science and technology

Scientific interest:

Process optimization and efficient use of energy

Publications $<$ number or main>: 20 Papers

\section{Experience:}

He received his Ph.D. degree from University of science and technology Beijing in 2001. Now he is deputy director of technical center of Tangshan Iron \& Steel. His current research areas are process optimization and efficient use of energy.

\section{Ying Tian,}

Current position,

Doctor of Computer Science, professor and supervisor of postgraduate in University of Science and Technology Liaoning

University studies:

Measurement technology and instrument in Shenyang University of Technology

Scientific interest:

Biometrics, Pattern recognition and Image processing.

Publications $<$ number or main $>$ : 20 Papers

Experience:

She received Ph.D degrees on Digital Image Processing from the Shenyang University of Technology, 2008. Now she is a professor at University of Science and Technology Liaoning, Anshan, Liaoning, China. She mainly focuses on pattern recognition and digital image processing.

\section{CONFLICT OF INTEREST}

The authors confirm that this article content has no conflict of interest.

\section{ACKNOWLEDGEMENTS}

This work was supported by scientific research fund of Liaoning provincial education department (L2014118) and the Anshan science and technology park.

\section{REFERENCES}

[1] R. Eberhart, J. Kennedy, "A Hew optimizer using particle swarm theory", Proceedings of the $6^{\text {th }}$ International Symposium on Micro Machine and Human Science, Nagoya, Japan, pp.39-43, 1995.

[2] J. Kennedy, R.C. Eberhart, "Particle swarm optimization", Proceedings of the 1995 IEEE International Conference on Neural Networks, New York, pp. 1942-1948, 1995.

[3] S. Farzi, "Training of fuzzy neural networks via quantum-behaved particle swarm optimization and rival penalized competitive learning", The International Arab Journal of Information Technology, vol. 9, no. 4, pp. 306-313, 2012.

[4] P.N. Suganthan, "Particle swarm optimizer with neighborhood operator", Proceedings of the IEEE Congress on Evolutionary Computation, Piscataway, NJ, pp. 1958-1962, 1999.

[5] A. Marco, Montes de Oca., S. Thomas, B. Mauro, D. "Marco, Frankenstein's PSO: a composite particle swarm optimization algorithm", IEEE Transactions on Evolutionary Computation, vol. 13, no. 5, pp.1120-1132, 2009

[6] N. Qing-Jian, X. Han-Cheng, Z. Zhi-Zheng, W. Zhen-Zhen, "A multi-cluster structure based gaussian dynamic particle swarm optimization algorithm", Pattern Recognition and Artificial Intelligence, vol. 21, no 3, pp.338-345, 2008. 
[7] Y. Xue-rong, L. Jia-hong, C. Ling, Y. Da-wei, "Multineighborhood improved particle swarm optimization algorithm", Systems Engineering and Electronics. vol. 32, no. 11, pp. 2453$2458,2010$.

[8] G. Ying, X. Sheng-li, X. Ruo-ning, L. Zhao-hui, "A multi subpopulation particle swarm optimizer based on clustering", Application Research of Computers, vol. 23, no. 4, pp. 40-41, 2006.

[9] W. Wen, H. Zhi-feng, "Improved particle swarm optimizer based on dynamic topology", Computer Engineering and Applications, vol. 41, no. 34, pp. 82-85, 2005.

[10] Y. Shi, R.C. Eberhart, "A modified particle swarm optimizer", Proceedings of the 1998 IEEE International Conference on Evolutionary Computation, Piscataway, USA, pp. 67-73, 1998.
[11] A. Ratnaweera, S.K. Halgamuge, H.C. Watson, "Self-organizing hierarchical particle swarm optimizer with time-varying acceleration coefficients", Evolutionary Computation, vol. 8, no. 3, pp.240-255, 2004.

[12] L. Xiao-lei, S. Zhi-jiang, Q. Ji-xin, "An optimizing method based on autonomous animats:fish-swarm algorithm", Systems Engineering-theory and Practice, vol. 22, no. 11, pp. 32-38, 2002.

[13] M. Clerc, J. Kennedy, "The particle swarm: explosion, stability, and convergence in a multi-dimensional complex space", IEEE Transactions on Evolutionary Computation, vol. 8, no. 6, pp. 58-73, 2004.

[14] Z. Hai-Mei, W. yong-ping, "A PSO algorithm with high speed convergence", Control and Decision, vol. 25, no 1, pp.20-30, 2010.

(C) Kai et al.; Licensee Bentham Open.

This is an open access article licensed under the terms of the Creative Commons Attribution Non-Commercial License (http://creativecommons.org/licenses/by-nc/3.0/) which permits unrestricted, non-commercial use, distribution and reproduction in any medium, provided the work is properly cited. 